\title{
Design of Low Noise Amplifier
}

\author{
Vinoth S \\ Dept. Of ECE \\ IIIT Hyderabad \\ India
}

\begin{abstract}
The value of capacitors and inductors placed are simulated based on characteristic equations derived from electron movements. In this paper, a statistical learner is used to help run the simulator.
\end{abstract}

Keywords: Statistical learner, low noise amplifier

\section{INTRODUCTION}

The value of capacitors and inductors placed in the input and output circuit of a low noise amplifier is simulated based on characteristic equations derived from electron movements.

\subsection{Theory}

Assume L1, C1 are placed in the input circuit and L2, C2 are placed in the output circuit. $\{(\mathrm{L} 1, \mathrm{C} 1),(\mathrm{L} 2, \mathrm{C} 2)\}$ pair creates a specific filter. This filter is used to learn the modulation of a low noise amplifier. The 2 nd pair is classified into four quantisation pairs while the 1st pair is clustered into 12 such pairs as quantised clusters. Output values are fixed as the output of the filter which are initiated as the signal filtered only from input, signal filtered only from output and difference of these two values differently by 2 numbers.

\subsection{Indentations and EQUATIONS}

$$
\begin{aligned}
& \text { Outputs: } \\
& 1 \text {-> signalfrom(L1,C1) } \\
& 2 \text {-> output4-output } 1 * 1 / 3 \\
& 3 \text {-> output4-output } 1 * 2 / 3 \\
& 4 \text {-> signalfrom(L2,C2) }
\end{aligned}
$$

Mathematically the proposed $1 \times 4$ sized output vector $\mathrm{O}=[\mathrm{g} 1 \mathrm{~g} 2 \mathrm{~g} 3 \mathrm{~g} 4]$ is related with $1 \times 5$ sized input vector $\mathrm{I}=$ [G1G2G3G4G5] (which are obtained from the arbitrary $3 \times 3$ sized green channel) in the

following manner. $\mathrm{H}=\mathrm{f} 1(\mathrm{I} \times \mathrm{W} 1+\mathrm{B} 1)$ and $\mathrm{O}=\mathrm{H} \times \mathrm{W} 2+\mathrm{B} 2$ where $\mathrm{H}$ is $1 \times 2$ sized hidden vector, $\mathrm{W} 1$ is $5 \times 2$ sized weight matrix (between input layer and hidden layer), B1 is $1 \times 2$ sized biased vector at hidden layer, W2 is $2 \times 4$ sized weight matrix (between hidden layer and output layer), B2 is $1 \times 4$ sized bias vector at the output layer. $\mathrm{f} 1$ is chosen as the nonlinear function $\log \operatorname{sig}(\mathrm{n})=1$

$\left.1+\exp (-)^{+}\right)$

\begin{abstract}
. Suppose the digital image captured using the digital camera is subjected to manipulation like rotation, resize, scaling etc. using the image edit tool boxes like photo shop and paint the relationship above gets distorted and is no more valid. The values of the above mentioned matrices and vectors (W1, B1, W2 and B2) are arranged in a single row to obtain a feature vector of size $1 \times 24$. Thus the feature vector of size $1 \times 24$ obtained as described helps in identifying whether the particular part of the image is original or forged. The proposed feature vector is [W1 (stacked in a single row) W2 (stacked in a single row) B1 (stacked in a single row) B2 (stacked in a single row)]
\end{abstract}

\section{Proposed forgery detector}

From each of the image, $5003 \times 3$ sub blocks were collected at random. $1 \times 5$ sized input vectors and the corresponding $1 \times 4$ sized output vectors were collected from every $3 \times 3$ sub blocks of the green channel matrix. In a Bayer array filter the number of green sensors is more than the number of red and blue sensors. So, in this paper we have used the green values taken from the image to model the neural network.

The feature vectors are obtained as mentioned in section 2 . This vector completely describes the interpolation elationship. The input and output vectors collected from these 500 sub blocks were used to train the BPN to get one feature vector of size $1 \times 24$. This is repeated for all the input output vectors from each image and this formed the feature vectors for the original images. Doctored images were created by copy paste, resize, rotation etc. and the input and output vectors were collected from the doctored part of the image and the above steps were repeated to extract feature vectors for the forged images. 
To check the accuracy with which the neural network has modeled the camera 50 such sub blocks were taken from the same image and the predicted outputs and the corresponding original values were plotted. Expected values are the values taken from the corresponding pixel of each image (interpolation done by the camera) and predicted values are those done by the trained neural network.

Dimensionality reduction, training the classifier and testing phase About 100 feature vectors were collected from both original and forged images. 50 such feature vectors (collected from 4 different cameras in case they were from original data set) of them were used for training. They were projected onto kernel space (7 different kernels were used) and LDA was performed to reduce their dimension from $1 \times 24$ to 1 . This one dimensional data was used to train our classifier. We used three different types of classifier: SVM, nearest neighbor and nearest mean. Five different kernels were used for SVM. The remaining 50 feature vectors were used for testing. Their dimensions were reduced from $1 \times 24$ to 1 as in the testing phase and were fed as inputs to the three different classifiers. The outputs are tabulated in Table 1.

\section{REFERENCES}

[1] Vinoth S, ES Gopi, Neural Network modeling of color array filter for image forgery detection using kernel LDA. . 